\title{
OUTER MEASURES ON A LINEAR LATTICE
}

\author{
BY \\ LEON BROWN AND HIDEGORO NAKANO(1)
}

In this paper we define and discuss the theory of abstract outer measures on a sequentially continuous $\left({ }^{2}\right)$ linear lattice $S$. This is a generalization of the concept of outer measure on a function space as used by Bourbaki [3]. H. Nakano [7] and M. H. Stone [8] have modernized Lebesgue's extension theory; our approach provides a common generalization of their theories. We show, for example, that their theories lead to the same set of integrable functions, and that, from a lattice theoretic point of view, Stone's results depend on the fact that the space of all real-valued functions on a set $X$ is a perfect( $\left.{ }^{3}\right)$ lattice.

I. Outer measures. In this section we define outer measure and we introduce a partial ordering for the space of outer measures. For a given outer measure $P^{*}$ we introduce a subspace $S_{P *}$ of $S$, which plays the role of the space of integrable elements.

Throughout this paper $S$ is a sequentially continuous linear lattice. $P^{*}$ is called an outer measure on $S$ if $P^{*}$ satisfies the following properties:

(a) $P^{*}$ is a function from $S$ into the extended reals $\left(-\infty \leqq P^{*}(x) \leqq+\infty\right)$;

(b) if $x \leqq y$ then $P^{*}(x) \leqq P^{*}(y)$;

(c) if $\alpha \geqq 0$ then $P^{*}(\alpha x)=\alpha P^{*}(x)(0 \cdot \infty=0(-\infty)=0)$;

(d) if $P^{*}(x)+P^{*}(y)$ is defined, then

$$
P^{*}(x+y) \leqq P^{*}(x)+P^{*}(y),
$$

and if in addition, $P^{*}(x \wedge y)+P^{*}(x \vee y)$ is defined, then

$$
P^{*}(x \vee y)+P^{*}(x \wedge y) \leqq P^{*}(x)+P^{*}(y) ;
$$

(e) if $x_{n}$ is a monotonically increasing sequence in $S, P^{*}\left(x_{1}\right)>-\infty$, and $x=\bigvee_{n} x_{n}$ then $P^{*}(x)=\lim _{n \rightarrow \infty} P^{*}\left(x_{n}\right)$.

We present some consequences of our axioms.

LEMMA 1.1. $-P^{*}(-x) \leqq P^{*}(x)$ for each $x \in S$.

Presented to the Society, January 25, 1963; received by the editors August 12, 1964.

(1) This work was done with the partial support of NSF Grant GP-54.

(2) A linear lattice $S$ is said to be sequentially continuous if for every sequence $\left\{a_{n}\right\}$ of positive elements, $\bigwedge a_{n}$ exists. This concept is called 6-completeness in [2, p. 80].

(3) A linear lattice $S$ is said to be perfect if for each sequence $\left[a_{n}\right] \subset S, \Sigma a_{n}$ converges provided that $a_{n} \wedge a_{m}=0$ for $n \neq m$. In the literature, various other terms are used for the concept of perfect such as complete [5, p. 155], and Volkommen [6]. 
Proof. If $P^{*}(x)+P^{*}(-x)$ is not defined then $P^{*}(x)= \pm \infty$ and $P^{*}(-x)=\mp \infty$. If it is defined, then $0=0 P^{*}(0)=P^{*}(0) \leqq P^{*}(x)+P^{*}(-x)$. q.e.d.

We write $P^{*} \geqq Q^{*}$ if $P^{*}(x) \geqq Q^{*}(x)$ for each $x$ in $S$.

With the above definition of $\geqq$, one easily shows that the collection of all outer measures forms a partially ordered set.

Putting $M^{*}(x)=+\infty$ for $x^{+} \neq 0$, and $=0$ for $x^{+}=0$ (i.e., $x \leqq 0$ ), we obtain an outer measure $M^{*}$, and we see easily $M^{*}$ is the greatest (maximum) outer measure.

We will make use of the notation

$$
S_{P^{*}}=\left\{x \mid P^{*}(|x|)<+\infty \text { and } P^{*}(x)=-P^{*}(-x)\right\} .
$$

Then we have

THEOREM 1.2. $S_{P^{*}}$ is a subspace of $S$ and $P^{*}$ restricted to $S_{P^{*}}$ is a positive linear functional which is sequentially continuous. $P^{*}$ is sequentially continuous if whenever $a_{n} \downarrow 0$ then $P^{*}\left(a_{n}\right) \rightarrow 0$.

Proof. We recall that a subspace of $S$ [Nakano 4, p. 14] is closed under finite sups and infs and if $x_{n}\left(x_{n} \geqq 0\right)$ is in the subspace for each $n$, then $\bigwedge_{n} x_{n}$ is in the subspace.

(a) If $x \geqq 0$ then $P^{*}(x) \geqq P^{*}(0)=0$;

(b) as $P^{*}(-x+(-y)) \leqq P^{*}(-x)+P^{*}(-y)$, for $x, y \in S_{P^{*}}$, we have $-P^{*}(-(x+y)) \geqq-P^{*}(-x)-P^{*}(-y)=P^{*}(x)+P^{*}(y) \geqq P^{*}(x+y)$. Thus we obtain by Lemma $1.1, x+y \in S_{P^{*}}$ and $P^{*}(x+y)=P^{*}(x)+P^{*}(y)$.

(c) Let $x \in S_{P^{*}}$. If $\alpha \geqq 0$, then

$$
P^{*}(\alpha x)=\alpha P^{*}(x)=-\alpha P^{*}(-x)=-P^{*}(-\alpha x) .
$$

If $\alpha<0$ then

$$
P^{*}(\alpha x)=P^{*}((-\alpha)(-x))=-\alpha P^{*}(-x)=\alpha P^{*}(x)=-(-\alpha) P^{*}(x)=-P^{*}(-\alpha x) .
$$

Thus $\alpha x \in S_{P^{*}}$ and $P^{*}(\alpha x)=\alpha P^{*}(x)$.

(d) Let $x, y \in S_{P^{*}}$. Since

$$
\begin{aligned}
P^{*}(x \wedge y) \leqq P^{*}(x \vee y) & \leqq P^{*}(|x| \vee|y|) \leqq P^{*}(|x|+|y|) \\
& \leqq P^{*}(|x|)+P^{*}(|y|)<+\infty
\end{aligned}
$$

we have by Lemma 1.1

$$
\begin{aligned}
P^{*}(x)+P^{*}(y) & =-\left(P^{*}(-x)+P^{*}(-y)\right) \\
& \leqq-\left(P^{*}(-x \vee-y)+P^{*}(-x \wedge-y)\right) \\
& =-\left(P^{*}(-(x \wedge y))+P^{*}(-(x \vee y))\right. \\
& \leqq P^{*}(x \wedge y)+P^{*}(x \vee y) \leqq P^{*}(x)+P^{*}(y)
\end{aligned}
$$


Thus $-P^{*}(-(x \wedge y))-P^{*}(-(x \vee y))=P^{*}(x \wedge y)+P^{*}(x \vee y)$. Since

$$
-P^{*}(-(x \wedge y)) \leqq P^{*}(x \wedge y), \text { and }-P^{*}(-(x \vee y)) \leqq P^{*}(x \vee y)
$$

by Lemma 1.1, we obtain $x \wedge y \in S_{P^{*}}$ and $x \vee y \in S_{P^{*}}$.

(e) Let $x_{n} \in S_{P^{*}}$ and $x_{n} \downarrow x$ in $S$. Then $-x_{n} \uparrow-x$ which implies that

$$
P^{*}\left(-x_{n}\right) \uparrow P^{*}(-x) \text {, that is, }-P^{*}\left(-x_{n}\right) \downarrow-P^{*}(-x) .
$$

As $P^{*}(x) \leqq P^{*}\left(x_{n}\right)=-P^{*}\left(-x_{n}\right)$, we obtain here $P^{*}(x) \leqq-P^{*}(-x) \leqq P^{*}(x)$ by Lemma 1.1. Therefore $P^{*}(x)=\lim _{n \rightarrow \infty} P^{*}\left(x_{n}\right)$ and $x \in S_{P^{*}}$. q.e.d.

II. Compatible outer measures. The relationship between positive linear functionals and outer measures is discussed. Of particular interest is Theorem 2.4, which implies that the theories of Nakano and Stone give rise to the same set of integrable functions. Let $C$ be a linear lattice manifold of $S$. A positive linear functional $P$ on $C$ is said to be compatible with an outer functional $P^{*}$ on $S$ if $P(x)=P^{*}(x)$ for all $x \in C$. We also say $P^{*}$ is compatible with $P$. If $P$ is compatible with $P^{*}$, then $C \subset S_{P^{*}}$, because $x \in C$ implies $-P^{*}(-x)=-P(-x)=P(x)=P^{*}(x)$.

THEOREM 2.1. Let $P$ be a positive linear functional on $C$ then there is a $P^{*}$ compatible with $P$ if and only if $P$ is sequentially continuous.

Proof. (a) If $P$ is sequentially continuous then let

$$
P^{*}(x)=\inf \left\{\lambda \mid \lambda=\lim P\left(a_{n}\right): C \ni a_{n} \uparrow, a_{n} \wedge x \uparrow x, \text { or } \lambda=+\cdots\right\} .
$$

Then $P^{*}$ is an outer measure compatible with $P$ (see [Nakano 7]).

(b) If $P^{*}$ is compatible with $P$ and $C \ni x_{n} \downarrow 0$, then $-x_{n} \uparrow 0$ and

$$
P\left(-x_{n}\right)=P^{*}\left(-x_{n}\right) \uparrow P^{*}(0)=0 \text {, namely } P^{*}\left(x_{n}\right) \downarrow 0 . \quad \text { q.e.d }
$$

THEOREM 2.2. If $P$ is a positive, sequentially continuous linear functional on $C$ then there is the greatest compatible $P^{*}$, and we have

$$
P^{*}(x)=\inf \left\{\lambda \mid \lambda=+\infty \text { or } \lambda=\lim P\left(a_{n}\right): C \ni:_{n} \uparrow, a_{n} \wedge x \uparrow x\right\} .
$$

Proof. Let $P_{1}^{*}$ be compatible with $P$ and $x \in S$. Then we have

(a) If $P^{*}(x)=+\infty$ then $P_{1}^{*}(x) \leqq P^{*}(x)$.

(b) If $P^{*}(x)=-\infty$ then given $\alpha$, there is $C \ni a_{n} \uparrow a_{n} \wedge x \uparrow x$ and $\lim _{n \rightarrow \infty} P\left(a_{n}\right)<\alpha$. Therefore $P_{1}^{*}(x)=\lim _{n \rightarrow \infty} P_{1}^{*}\left(a_{n} \wedge x\right) \leqq \lim _{n \rightarrow \infty} P_{1}^{*}\left(a_{n}\right)=\lim _{n \rightarrow \infty} P\left(a_{n}\right)<\alpha$.

(c) If $\left|P^{*}(x)\right|<+\infty$, then for any $\varepsilon>0$, there is $C \ni a_{n} \uparrow, a_{n} \wedge x \uparrow x$ such that $P^{*}(x)+\varepsilon \geqq \lim _{n \rightarrow \infty} P\left(a_{n}\right)$.

Thus $P_{1}^{*}(x)=\lim _{n \rightarrow \infty} P_{1}^{*}\left(a_{n} \wedge x\right) \leqq \lim _{n \rightarrow \infty} P\left(a_{n}\right) \leqq P^{*}(x)+\varepsilon$. Therefore $P_{1}^{*}(x) \leqq P^{*}(x)$. q.e.d.

LEMMA 2.3. If $P^{*}$ is an outer measure on $S$ and if $y \in S_{P^{*}}$ then 


$$
P^{*}(x+y)=P^{*}(x)+P^{*}(y) .
$$

Proof. $P^{*}(x)+P^{*}(y)=P^{*}(x)-P^{*}(-y) \leqq P^{*}(x+y) \leqq P^{*}(x)+P^{*}(y)$. q.e.d.

THEOREM 2.4. If $P$ is a positive, sequentially continuous, linear functional on $C$, and $P^{*}$ is the greatest outer measure compatible with $P$, then $x \in S_{P *}$ if and only if there is a sequence $a_{n} \in C$ such that $\lim _{n \rightarrow \infty} P^{*}\left(\left|x-a_{n}\right|\right)=0$.

Proof. (a) Let $a_{n} \in C$ and $\lim _{n \rightarrow \infty} P^{*}\left(\left|x-a_{n}\right|\right)=0$. Then

$$
\left|P^{*}(x)-P^{*}\left(a_{n}\right)\right|=\left|P^{*}(x)+P^{*}\left(-a_{n}\right)\right|=\left|P^{*}\left(x-a_{n}\right)\right| \leqq P^{*}\left(\left|x-a_{n}\right|\right)
$$

and

$$
\left|P^{*}(-x)+P^{*}\left(a_{n}\right)\right|=\left|P^{*}\left(a_{n}-x\right)\right| \leqq P^{*}\left(\left|a_{n}-x\right|\right)=P^{*}\left(\left|x-a_{n}\right|\right) .
$$

Thus $-P^{*}(-x)=\lim _{n} \downarrow{ }_{\infty} P^{*}\left(a_{n}\right)=P^{*}(x)$ and $x \in S_{P}$.

(b) Conversely let $x \in S_{P^{*}}$; for any $\varepsilon>0$ there is a sequence $a_{n} \in C$ such that $a_{n} \uparrow, a_{n} \wedge x \uparrow x$ and $\lim _{n \rightarrow \infty} P\left(a_{n}\right) \leqq P^{*}(x)+\varepsilon / 2$. Then

$$
\begin{aligned}
P^{*}\left(\left|x-a_{n}\right|\right) & =P^{*}\left(\left(x-a_{n}\right)^{+}+\left(a_{n}-x\right)^{+}\right) \\
& =P^{*}\left(\left(x-a_{n}\right)^{+}\right)+P^{*}\left(\left(a_{n}-x\right)^{+}\right) \\
& =P^{*}\left(x-x \wedge a_{n}\right)+P^{*}\left(a_{n}-a_{n} \wedge x\right) \\
& =P^{*}(x)-P^{*}\left(x \wedge a_{n}\right)+P^{*}\left(a_{n}\right)-P^{*}\left(a_{n} \wedge x\right) .
\end{aligned}
$$

Therefore $\lim _{n \rightarrow \infty} P^{*}\left(\left|x-a_{n}\right|\right)=P^{*}(x)-P^{*}(x)+\lim _{n \rightarrow \infty} P^{*}\left(a_{n}\right)-P^{*}(x) \leqq \varepsilon / 2$. Therefore, there exists an $n$ such that $P^{*}\left|x-a_{n}\right|<\varepsilon$. q.e.d.

THEOREM 2.5. If $P$ is a positive, sequentially continuous, linear functional on $C$, and $P^{*}$ is the greatest outer measure compatible with $P$, then the following are equivalent:

(i) $P^{*} \geqq Q^{*}$ where $Q^{*}$ is an outer measure,

(ii) $S_{P^{*}} \subset S_{Q^{*}}$ and $P^{*}(x)=Q^{*}(x)$ for $x \in S_{P^{*}}$,

(iii) $C \subset S_{Q^{*}}$ and $P(x)=Q^{*}(x)$ for $x \in C$.

Proof. (i) $\Rightarrow$ (ii): If $P^{*} \geqq Q^{*}$ and $x \in S_{P^{*}}$, then $P^{*}(x)-=P^{*}(-x) \leqq$ $-Q^{*}(-x) \leqq Q^{*}(x) \leqq P^{*}(x)$ and $Q^{*}(|x|) \leqq P^{*}(|x|)<+\infty$. Thus $x \in S_{Q^{*}}$ and $P^{*}(x)=Q^{*}(x)$.

(ii) implies (iii): because $C \subset S_{P *}$.

(iii) implies (i): since $P^{*}$ is the greatest outer measure compatible with $P$.q.e.d. For a manifold $C \subset S$, let $\left.P^{*}\right|_{c}$ denote a functional $R$ on $C$ such that $R(x)=P^{*}(x)$ for $x \in C$. With this notation we have

COROLlaRy 2.6. Let $P^{*}$ be the greatest outer measure compatible with $P$. If $P_{1}=\left.P^{*}\right|_{S_{P^{*}}}$ and $P_{1}^{*}$ is the greatest outer measure compatible with $P_{1}$ then $P_{1}^{*}=P^{*}$. 
Proof. (a) $P_{1}^{*} \geqq P^{*}$ since $P^{*}$ is compatible with $P_{1}$.

(b) $P_{1}^{*} \leqq P^{*}$ since $S_{P^{*}} \subset S_{P_{1}^{*}}$ and $P_{1}^{*}(x)=P^{*}(x)$ for $x \in S_{P^{*} \text {. q.e.d. }}$

III. Regular outer measures. Although the collection of all outer measures is not a lattice, the set of regular outer measures greater than or equal to a fixed regular outer measure turns out to be a universally continuous lattice (that is, every set of elements has an infimum).

$P^{*}$ is called a regular outer measure if $P^{*}$ is the greatest outer measure compatible with $P^{*} \mid S_{P^{*}}$.

Corollary 2.6 says that $P^{*}$ is a regular outer measure if and only if $P^{*}$ is the greatest extension of some positive, sequentially continuous, linear functional.

EXAMPLE 3.1. If $S$ is the space of real numbers and $P^{*}(x)=x \chi_{[0, \infty]}(x)$ then $P^{*}$ is a nonregular outer measure. Note that $S_{P^{*}}=\{0\}$ and this induces $M^{*}>P^{*}$.

In this section all outer measures will be regular outer measures.

Lemma 3.2. If $P^{*} \wedge Q^{*}$ exists, then $P^{*}(x)=Q^{*}(x)$ for $x \in S_{P^{*}} \cap S_{Q^{*}}$

Proof. The result follows from Theorem 2.5, because if $R^{*}=P^{*} \wedge Q^{*}$ then $R^{*} \leqq P^{*}$ and $R^{*} \leqq Q^{*}$.

Lemma 3.3. Let $C=\left\{x \mid x \in S_{P^{*}} \cap S_{Q^{*}}\right.$ and $\left.P^{*}(x)=Q^{*}(x)\right\}$. If $C$ is a subspace of $S$ then $P^{*} \vee Q^{*}$ exists and $S_{P^{*} \vee Q}=C$.

Proof. If $R=\left.P^{*}\right|_{c}$, then $R$ is a positive, sequentially continuous, linear functional on $C$. Let $R^{*}$ be the greatest outer measure compatible with $R$. Since $P^{*}$ and $Q^{*}$ are compatible with $R, P^{*} \leqq R^{*}$ and $Q^{*} \leqq R^{*}$. If $R_{1}^{*} \geqq P^{*}$ and $\geqq Q^{*}$, then $S_{R_{1}^{*}} \subset C$ since if $x \in S_{R_{i}^{*}}$ then $x \in S_{P^{*}} \cap S_{Q^{*}}$ and $R_{1}^{*}(x)=P^{*}(x)=Q^{*}(x)$. Therefore $R_{1}^{*} \geqq R^{*}$ and $S_{R_{1}^{*}} \subset C \subset S_{R^{*}}$ which implies $S_{R^{*}}=C$. q.e.d.

We present an example of two regular outer measures that have no infimum in the space of all outer measures (nor in the space of all regular outer measures), although they have a supremum.

EXAMPLE 3.4. Let $S=\{f \mid f:[0,1] \rightarrow$ reals $\}=$ (Reals) $[0,1]$.

$$
C_{0}=\left\{f \mid f=a \chi_{A}+b \chi_{B}: A \cap B=\varnothing, A \neq \varnothing, B \neq \varnothing\right\},
$$

$P\left(a \chi_{A}+b \chi_{B}\right)=a+2 b$, and $Q\left(a \chi_{A}+b \chi_{B}\right)=2 a+3 b$. One can easily show that $S_{P^{*}}=S_{Q^{*}}=C_{0}$ and $P(f)=Q(f)$ iff $a=-b$. Therefore $C=\left\{f \mid f=a\left(\chi-\chi_{B}\right)\right\}$ which is not a lattice since $\left(\chi_{A}-\chi_{B}\right) \vee\left(\chi_{B}-\chi_{A}\right)=\chi_{A}+\chi_{B} \notin C$. Also if $R^{*} \geqq P^{*}$ and $\geqq Q^{*}$ then $S_{R^{*}} \leqq C$ and we have that $S_{R^{*}}=\{0\}$ and $R^{*}=M^{*}$ i.e., $P^{*} \vee Q^{*}$ $=M^{*}$.

REMARK. If $C=S_{P^{*}} \cap S_{Q^{*}}$ then $P^{*} \vee Q^{*}$ exists.

LEMma 3.5. If $P^{*} \wedge Q^{*}$ exists then $P^{*} \vee Q^{*}$ exists.

Proof. If $P^{*} \wedge Q^{*}$ exists then Lemma 3.2 states that $C=S_{p^{*}} \cap S_{Q^{*} \text {. q.e.d. }}$ 
THEOREM 3.6. Let $P_{0}^{*}$ be a regular outer measure. Then $H=\left\{P^{*} \mid P^{*} \geqq P_{0}^{*}\right\}$ is a universally continuous lattice.

Proof. (I) $H$ is a lattice. Let $P_{1}^{*}$ and $P_{2}^{*} \in H$, then $P_{1}^{*} \geqq P_{0}^{*}$ and $P_{2}^{*} \geqq P_{0}^{*}$ which implies that $S_{P_{1}^{*}} \cup S_{P_{2}^{*}} \subset S_{P_{0}^{*}}$ and if $x \in S_{P_{i}^{*}}, i=1,2$, then $P_{i}^{*}(x)=P_{0}^{*}(x)$.

If $Q=\bigcap\left\{Q_{\lambda} \mid Q_{\lambda} \supset S_{P_{1}^{*}} \cup S_{P_{2}^{*}}\right.$ and $Q_{\lambda}$ is a subspace $\}$, then $Q$ is a subspace and $Q \subset S_{P_{0}^{*}}$. Let $P=\left.P_{0}^{*}\right|_{Q}$ and $P^{*}$ the greatest outer measure compatible with $P$. Then $S_{P^{*}} \supset Q \supset S_{P_{1}^{*}} \cup S_{P_{2}^{*}}$ and $P^{*}(x)=P_{0}^{*}(x)=P_{i}^{*}(x)$ on $S_{P_{i}^{*}}$. Therefore $P^{*} \leqq P_{1}^{*}$ and $P^{*} \leqq P_{2}^{*}$.

If $P_{3}^{*} \in H, P_{3}^{*} \leqq P_{1}^{*}, P_{3}^{*} \leqq P_{2}^{*}$ then $S_{P_{3}^{*}} \supset S_{P^{*}} \cup S_{P_{2}^{*}}$ which implies that $S_{P_{3}^{*}} \supset Q$ and $P_{3}^{*}(x)=P_{0}^{*}(x)=P^{*}(x)=P(x)$ on $Q$. Thus $P_{3}^{*}$ is compatible with $P$ and we have $P_{3}^{*} \leqq P^{*}$.

We now have that $P_{1}^{*} \wedge P_{2}^{*}=P^{*}$ exists and by Lemma $3.5, P_{1}^{*} \vee P_{2}^{*}$ exists and is clearly in $H$.

(II) $H$ is universally continuous.

Let $\left\{P_{\lambda}^{*} \mid \lambda \in I\right\} \subset H$. Then $\bigvee_{\lambda \in I} P_{\lambda}^{*}$ and $\bigwedge_{\lambda \in I} P_{\lambda}^{*}$ exists. Since $Q=\bigcap_{\lambda \in I} S_{P_{\lambda}^{*}} \supset\{0\}$, is a subspace we let $P=\left.P_{0}^{*}\right|_{Q}$ and $P^{*}$ the greatest outer measure compatible with $P$. Each $P_{\lambda}^{*}$ is compatible with $P$ which implies $P_{\lambda}^{*} \leqq P^{*}$ for all $\lambda \in I$. If $P_{\lambda}^{*} \leqq P_{1}^{*}$ for all $\lambda \in I$, then $S_{P_{1}^{*}} \subseteq \bigcap_{\lambda \in I} S_{P_{\lambda}^{*}}=Q$, and $P_{1}^{*}(x)=P_{\lambda}^{*}(x)=P_{0}^{*}(x)=P(x)$ for $x \in S_{P_{1}^{*}}$. Therefore $P^{*} \leqq P_{1}^{*}$ and in fact $S \bigwedge_{\lambda \in I} P_{\lambda}^{*}=\bigcap_{\lambda \in I} S_{P^{*} \lambda} \cdot \bigwedge_{\lambda \in I} P_{\lambda}^{*}=\bigvee\left\{P^{*} \mid H \ni P^{*} \leqq P_{\lambda}^{*}\right.$ for all $\lambda \in I\}$. Note $P_{0}^{*} \leqq P_{\lambda}^{*}$ for all $\lambda \in I$. q.e.d.

IV. Known results on projectors and normal manifolds. A subset $N$ of $S$ is said to be a normal manifold if for each $a \in S$, there exists $x \in N$ and a $y \in N^{\perp}$ such that $a=x+y$.

One easily proves that $x$ and $y$ are unique and $N=N^{\perp \perp}$. We can define the projection operator [N] as follows: If $N$ is a normal manifold, then $[N] a=x$ where $a=x+y, x \in N$ and $y \in N^{\perp}$. If $M$ is a subset of $S, M$ is said to be normable if $M^{\perp}$ is a normal manifold and in this case we define $[M]=\left[M^{\perp \perp}\right]$. We write $[M] \geqq[N]$ iff $[M] x \geqq[N] x$ for all $x \geqq 0$. If $p \in S$ and $\{p\}$ is normable then $[p]$ is called a projector.

The next two theorems are summaries of a few results on projection operators and projectors presented in Nakano's book [4, pp. 14-28].

THEOREM 4.1. Let $N, K$, and $M$ be normable manifolds, then

(a) $[N]$ is a positive, linear, and idempotent operator,

(b) $|[N] a|=[N]|a| \leqq|a|$,

(c) $[N] a=a$ iff $a \in N$, and $[N] a=0$ iff $a \in N^{\perp}$,

(d) $[N]+\left[N^{\perp}\right]=1$,

(e) $[N](a \wedge b)=([N] a) \wedge b=a \wedge([N] b)$ for $a, b \geqq 0$,

(f) $[N]$ is continuous: i.e., $\lim _{n \rightarrow \infty} a_{n}=a$, implies $\lim _{n \rightarrow \infty}[N] a_{n}=[N] a$,

(g) $K \perp M$ iff $[K][M]=0$, 
(h) $[K]+[M]$ is a projection operator iff $[K][M]=0$, and in this case $[K]+[M]=[K, M]=[K \cup M]$.

In a sequentially continuous linear lattice every element $p$ is normable. We state the following theorem for the special case when $S$ is sequentially continuous

THEOREM 4.2. Let $p, q, a \in S$, then

(a) $[p]=[\alpha p]$ if $\alpha \neq 0$,

(b) $\left[a^{+}\right] a=a^{+}$and $\left(1-\left[a^{+}\right]\right) a=-a^{-}$,

(c) $|p| \geqq|q|$ implies $[p] \geqq[q]$,

(d) $[p] \geqq[q]$ iff $[p] q=q$,

(e) $[p] \geqq[q]$ implies $[p]-[q]=[(1-[q]) p]$,

(f) $[p] a=\bigvee_{n}(a \wedge n|p|)$ for $a \geqq 0$,

(g) $[p] \wedge[q]=[p][q]=[|p| \wedge|q|]$ and $[p] \vee[q]=[|p| \vee|q|]$ $=[|p|+|q|]$,

(h) $[p+q]=[p]+[q]$ iff $p \perp q$,

(i) $0 \leqq p_{n} \uparrow p$ implies $\left[p_{n}\right] \uparrow[p]$,

(j) for any $p_{n} \in S(n=1,2, \cdots), \wedge\left[p_{n}\right]$ exists and $\left(\bigwedge_{n=1}^{\infty}\left[p_{n}\right]\right) a=\bigwedge_{n=1}^{\infty}\left[p_{n}\right] a$ for $a \geqq 0$,

(k) given a sequence of projectors $\left[p_{n}\right]$ such that there exists a projector $\left[p_{0}\right] \geqq\left[p_{n}\right]$ for every $n$ then $\bigvee_{n=1}^{\infty}\left[p_{n}\right]$ exists and $\left(\bigvee_{n=1}^{\infty}\left[p_{n}\right]\right) a=\bigvee_{n=1}^{\infty}\left(\left[p_{n}\right] a\right)$ for $a \geqq 0$.

We have need of one more result. Let $S$ be a normed linear lattice. $S$ is said to be monotone complete if $0 \leqq a_{n} \uparrow$ and $\sup _{n}\left\|a_{n}\right\|<+\infty$ implies $\bigvee_{n=1}^{\infty} a_{n}$ exists.

THEOREM 4.3 (AMEMIYA [1]). If $S$ is a monotone complete linear latice, then $S$ is complete; i.e., $S$ is a Banach space.

One should note that there exists spaces which are complete but not monotone complete.

V. Banach spaces induced by outer measures. The main result of this section is that if $P^{*}$ is an outer measure and $S$ is perfect, then $\hat{S} / N_{P^{*}}$ is monotone complete and thus a Banach space. Conversely, if $\hat{S} / N_{P^{*}}$ is complete for every regular outer measure, then $S$ is perfect. Thus we see that from a lattice theoretic point of view Stone's result depends on the fact that the space of all real-valued functions on a set $X$ is a perfect linear lattice.

In this section $P^{*}$ is assumed to be an outer measure which is not necessarily regular. We will make use of the notation $N_{P^{*}}=\left\{x \mid P^{*}(|x|)=0\right\}$.

THEOREM 5.1. $\quad N_{P^{*}}$ is a closed seminormal manifold of $S$.

Proof. (a) For $x, y \in N_{p *}$, we have

$$
0 \leqq P^{*}(|\alpha x+\beta y|) \leqq|\alpha| P^{*}(|x|)+|\beta| P^{*}(|y|)=0 \text {. }
$$


Thus $x, y \in N_{P *}$ implies $\alpha x+\beta y \in N_{P^{*}}$.

(b) If $x \in N_{P^{*}}$ and $|y| \leqq|x|$ then $0 \leqq P^{*}(|y|) \leqq P^{*}(|x|)=0$.

Thus $N_{P^{*}}$ is a seminormal manifold and thus a linear lattice manifold and in fact a subspace.

(c) If $0 \leqq x_{n} \uparrow x, x_{n} \in N_{p *}$ then $P^{*}(x)=\lim _{n \rightarrow \infty} P^{*}\left(x_{n}\right)=0$ and $x \in N_{P * \text {. q.e.d. }}$

LEMMA 5.2. $P^{*}(x)=0$ for $x \in N_{p * *}$

Proof. If $x \geqq 0$ then $P^{*}(-x)=0$ since $0 \leqq-P^{*}(-x) \leqq P^{*}(x)=0$. In the general case $-|x| \leqq x \leqq|x|$. q.e.d.

LEMMA 5.3 (NAKANO [3]). If $S$ is a sequentially continuous linear lattice and $H$, a closed seminormal manifold $[4, p$. 14] then $S / H$ is a sequentially continuous linear lattice.

For any element $x \in S$, the element of $S / H$ containing $x$ is denoted by $x+H=\hat{x}$.

LEMMA 5.4. $y \in x+N_{P *}$ implies $P^{*}(x)=P^{*}(y)$.

Proof. $P^{*}(y) \leqq P^{*}(y-x)+P^{*}(x) \leqq P^{*}(|y-x|)+P^{*}(x)=P^{*}(x)$.

Similarly $P^{*}(x) \leqq P^{*}(y)$, since $y \in x+H$ implies $x \in y+H$. q.e.d.

By means of Lemma 5.4 we can define

$$
P^{*}\left(x+N_{P^{*}}\right)=P^{*}(x) \text {. }
$$

We also define $\|x\|=P^{*}(|x|)$ for $x \in S$. Note that this is a pseudo-norm on $S=\{x \in S \mid\|x\|<+\infty\}$. Then we have obviously:

LEMMA 5.5. $N_{P^{*}}=\{x \mid\|x\|=0\}$ and $S / N_{P^{*}}$ is a normed linear space.

THEOREM 5.6. Let $L$ be a sequentially continuous normed linear lattice. If $0 \leqq a_{n} \uparrow a$ implies $\left\|a_{n}\right\| \uparrow\|a\|$, and if $a_{n} \wedge a_{m}=0$ for $n \neq m$, and

$$
\sup _{m}\left\|\sum_{n=1}^{m} a_{n}\right\|<+\infty,
$$

implies that $\Sigma a_{n}$ converges in $L$, then $L$ is monotone complete.

Proof. We will prove Theorem 5.6 in seven steps.

(1) Given $a_{n} \uparrow$ such that $\sup \left\|a_{n}\right\| \leqq M$, then there is $p$ such that $\left[a_{n}\right] \uparrow[p]$.

Proof of (1). For $n<m$ we have

$$
\begin{aligned}
\left(\left[a_{n}\right]-\right. & {\left.\left[a_{n-1}\right]\right)\left(\left[a_{m}\right]-\left[a_{m-1}\right]\right) } \\
& =\left[a_{n}\right]\left[a_{m}\right]-\left[a_{n}\right]\left[a_{m-1}\right]-\left[a_{n-1}\right]\left[a_{m}\right]+\left[a_{n-1}\right]\left[a_{m-1}\right] \\
& =\left[a_{n}\right]-\left[a_{n}\right]-\left[a_{n-1}\right]+\left[a_{n-1}\right]=0 .
\end{aligned}
$$

A similar computation for $n>m$ leads to the fact that if $n \neq m$ then 


$$
\left(\left[a_{n}\right]-\left[a_{n-1}\right]\right) a_{n} \perp\left(\left[a_{m}\right]-\left[a_{m-1}\right]\right) a_{m}
$$

Now

$$
\begin{aligned}
\left\|\sum_{n=1}^{m}\left(\left[a_{n}\right]-\left[a_{n-1}\right]\right) a_{n}\right\| & =\left\|\sum_{n=1}^{m} a_{n}-\left[a_{n-1}\right] a_{n}\right\| \\
& \leqq\left\|\sum_{n=1}^{m} a_{n}-a_{n-1}\right\|=\left\|a_{m}\right\| \leqq M .
\end{aligned}
$$

By hypothesis $\sum_{n=1}^{\infty}\left(\left[a_{n}\right]-\left[a_{n-1}\right]\right) a_{n}$ converges to some $p \geqq 0$. Since

$$
\begin{aligned}
{\left[\sum_{n=1}^{m}\left(\left[a_{n}\right]-\left[a_{n-1}\right] a_{n}\right)\right] } & =\sum_{n=1}^{m}\left(\left[a_{n}\right]-\left[a_{n-1}\right]\right)\left[a_{n}\right] \\
& =\sum_{n=1}^{m}\left[a_{n}\right]-\left[a_{n-1}\right]=\left[a_{m}\right],
\end{aligned}
$$

we obtain $\left[a_{m}\right] \uparrow[p]$.

(2) Putting $p_{m}=\bigwedge_{n}\left(m p-a_{n}\right)^{+}$, we have $\left[p_{m}\right] \uparrow$ and $\left[p_{m}\right] \leqq[p]$. Therefore $\bigvee\left[p_{m}\right]=[r] \leqq[p]$ for some $r$.

Proof of (2). Since

we have

$$
\left(m p-a_{n}\right)^{+} \leqq(m p)^{+}=m p
$$

$$
\left[p_{m}\right] \leqq\left[\left(m p-a_{n}\right)^{+}\right] \leqq[m p]=[p] .
$$

(3) $\bigvee_{n}\left[p_{m}\right] a_{n}=b_{m}$ exists and $b_{m} \leqq\left[p_{m}\right](m p)$.

Proof of (3). Since $\left[\left(m p-a_{n}\right)^{+}\right]\left(m p-a_{n}\right)=\left(m p-a_{n}\right)^{+} \geqq 0,\left[\left(m p-a_{n}\right)^{+}\right] m p$ $\geqq\left[\left(m p-a_{n}\right)^{+}\right] a_{n}$.

Since $\left[p_{m}\right] \leqq\left[\left(m p-a_{n}\right)^{+}\right],[p m]\left[\left(m p-a_{n}\right)^{+}\right]=\left[p_{m}\right]$. Thus $\left[p_{m}\right] m p \geqq\left[p_{m}\right] a_{n}$ for $n=1,2, \cdots$ and (3) follows.

(4) Putting $p_{0}=0, \sum_{k=1}^{\infty}\left(\left[p_{k}\right]-\left[p_{k-1}\right]\right) b_{k}=b$ converges.

Proof of (4).

$$
\begin{aligned}
{\left[p_{m}\right] a_{n} } & =\sum_{k=1}^{m}\left(\left[p_{k}\right]-\left[p_{k-1}\right]\right) a_{n} \\
& =\sum_{k=1}^{m}\left(\left[p_{k}\right]\left[p_{k}\right]-\left[p_{k-1}\right]\left[p_{k}\right]\right) a \\
& =\sum_{k=1}^{m}\left(\left[p_{k}\right]-\left[p_{k-1}\right]\right)\left[p_{k}\right] a_{n} \\
& \uparrow_{n} \sum_{k=1}^{m}\left(\left[p_{k}\right]-\left[p_{k-1}\right]\right) b_{k} \text { by (3). }
\end{aligned}
$$

Since $\left\|\left[p_{m}\right] a_{n}\right\| \leqq\left\|a_{n}\right\| \leqq M$, we have $\left\|\Sigma_{k=1}^{m}\left(\left[p_{k}\right]-\left[p_{k-1}\right]\right) b_{k}\right\| \leqq M$. As in the 
proof of (1) one easily shows that $\left\{\left(\left[p_{k}\right]-\left[p_{k-1}\right]\right) b_{k}\right\}$ is an orthogonal family. Therefore $\sum_{k=1}^{\infty}\left(\left[p_{k}\right]-\left[p_{k-1}\right]\right) b_{k}$ converges.

(5) $b_{n} \leqq b$.

Proof of (5). Since

$$
\begin{aligned}
b_{m} & =\lim _{k \rightarrow \infty}\left[p_{m}\right] a_{k} \\
& =\lim _{k \rightarrow \infty}\left[p_{m}\right]^{2} a_{k} \\
& =\left[p_{m}\right] \lim _{k \rightarrow \infty}\left[p_{m}\right] a_{k} \\
& =\left[p_{m}\right] b_{m}
\end{aligned}
$$

and

$$
\begin{aligned}
b_{m-1} & =\lim _{k \rightarrow \infty}\left[p_{m-1}\right] a_{k} \\
& =\lim _{k \rightarrow \infty}\left[p_{m-1}\right]\left[p_{m}\right] a_{k} \\
& =\left[p_{m-1}\right] \lim _{k \rightarrow \infty}\left[p_{m}\right] a_{k} \\
& =\left[p_{m-1}\right] b_{m} .
\end{aligned}
$$

We have

$$
\sum_{m=1}^{n}\left(\left[p_{m}\right]-\left[p_{m-1}\right] b_{m}=\sum_{m=1}^{n}\left(b_{m}-b_{m-1}\right)=b_{n} .\right.
$$

Therefore $b_{n} \leqq b$.

(6) $[r]=[p]$.

Proof of (6). Let $[q]=[p]-[r], \quad([r] \leqq[p])$. Since

$$
\left(I-\left[\left(m p-a_{n}\right)^{+}\right]\right)\left(m p-a_{n}\right)=-\left(m p-a_{n}\right)^{-} \leqq 0,
$$

we have $\left(I-\left[\left(m p-a_{n}\right)^{+}\right]\right) m p \leqq\left(I-\left[\left(m p-a_{n}\right)^{+}\right]\right) a_{n} \leqq a_{n}$.

Therefore $m\left\|[q]\left(I-\left[\left(m p-a_{n}\right)^{+}\right]\right) p\right\| \leqq\left\|a_{n}\right\| \leqq M$.

Since $\left(1-\left[\left(m p-a_{n}\right)^{+}\right]\right) \uparrow_{n}\left(1-\left[p_{m}\right]\right)$, we obtain

$$
m\left\|[q]\left(I-\left[p_{m}\right]\right) p\right\| \leqq M ;
$$

therefore $m\|[q] p\| \leqq M$ for all $m$, because $[q]\left[p_{m}\right]=0$ for all $m$. Consequently $\|[q] p\|=0$, that is, $[q] p=0$ or $p-[r] p=0$ which implies $[p] \leqq[r]$. Since $[r] \leqq[p]$ we have $[p]=[r]$.

One is now able to complete the proof of this theorem.

One has $\left[p_{m}\right] a_{n} \leqq b_{m} \leqq b$. Therefore $\lim _{b \geqq m \rightarrow \infty}\left[p_{m}\right] a_{n}=[r] a_{n}=[p] a_{n}=a_{n}$. Thus $\vee a_{n}$ exist. q.e.d. 
THEOREM 5.10. Let $P^{*}$ be an outer measure on $S$. Then if $S$ is perfect: (for a sequence $a_{n} \in S$ such that $a_{n} \wedge a_{m}=0$ for $n \neq m, \sum a_{n}$ converges) then $\hat{S} / N_{P *}$ satisfies the condition that for a sequence $\hat{a}_{n} \in S / N_{p^{*}}$ if $\hat{a}_{n} \wedge \hat{a}_{m}=0$ for $n \neq m$ and

$$
\sup _{m}\left\|\sum_{n=1}^{m} \hat{a}_{n}\right\|<+\infty
$$

then $\sum_{n=1}^{\infty} \hat{a}_{n}$ converges. Consequently $\hat{S} / N_{P *}$ is monotone complete and thus a Banach space. Note: $\hat{a}_{n}=a_{n}+N_{P^{*}}$.

Proof. (a) For $a, b \geqq 0$ we have $(b-[a \wedge b] b) a=((I-[a \wedge b]) b) \wedge a$ $=(I-[a \wedge b])(b \wedge a)=0$.

(b) If $a \in N_{P^{*}}$ then $\{a\}^{\perp \perp} \subset N_{P^{*}}$ (i.e., $[a] b \in N_{P^{*}}$ for all $b \in S$ ).

Proof of (b). If $b \geqq 0$ then

$$
\begin{aligned}
0 \leqq P^{*}([a] b) & =P^{*}\left(\lim _{m \rightarrow \infty}(m|a| \wedge b)\right) \\
& =\lim _{m \rightarrow \infty} P^{*}(m|a| \wedge b) \\
& \leqq \lim _{m \rightarrow \infty} P^{*}(m|a|)=0 .
\end{aligned}
$$

Let $\hat{a}_{n}$ be given and $0 \leqq a_{n}$ representives of $\hat{a}_{n}$. By induction one defines $b_{n}$ in the following manner:

$$
\begin{aligned}
& b_{1}=a_{1}, \\
& b_{n}=a_{n}-\left[a_{n} \wedge\left(\bigvee_{i=1}^{n-1} b_{i}\right)\right] a_{n} .
\end{aligned}
$$

One easily sees by (a) that $b_{n} \geqq 0$ and $b_{n} \perp \bigvee_{i=1}^{n-1} b_{i}$ and thus $b_{n} \perp b_{i}$ for $i<n$.

We need to prove $\hat{b}_{n}=\hat{a}_{n}$. $\hat{b}_{1}=\hat{a}_{1}$ is clear. Suppose $\hat{b}_{i}=\hat{a}_{i}$ for all $i<n$ then since $\hat{a}_{n} \perp \bigvee_{i=1}^{n-1} \hat{a}_{i}=\bigvee_{i=1}^{n-1} \hat{b}_{i}, P^{*}\left(a_{n} \wedge\left(\bigvee_{i=1}^{n-1} b_{i}\right)\right)=0$ which implies by (b) that $P^{*}\left(\left[a_{n} \wedge \bigvee_{i=1}^{n-1} b_{i}\right] a_{n}\right)=0$ and thus $\hat{b}_{n}=\hat{a}_{n}$. Since $S$ is perfect $\sum_{n=1}^{\infty} b_{n}$ converges to $b$ and consequently $\sum_{n=1}^{m} b_{n} \uparrow_{n} b$. Thus

$$
P^{*}(b)=\sup _{m} P^{*}\left(\sum_{n=1}^{m} a_{n}\right)=\sup _{m}\left\|_{n=1}^{m} \hat{a}_{n}\right\|<+\infty \text {. }
$$

Therefore $b \in \hat{S}$ and $\sum_{n=1}^{\infty} \hat{a}_{n}=\hat{b}$.

(c) If $0 \leqq \hat{a}_{n} \uparrow \hat{a}$ then $\lim _{n \rightarrow \infty}\left\|\hat{a}_{n}\right\|=\|\hat{a}\|$.

Proof of (c). We may assume that $a_{n} \leqq a$ for all $n$. If $b_{n}=\mathbb{I} \vee_{i=1}^{n} a_{i}, b_{n} \uparrow a_{0}$, then $\hat{b}_{n}=\hat{a}_{n}, \quad \hat{a}_{0}=\hat{a}$, and thus $\|(\hat{a})\|=P^{*}\left(a_{0}\right)=\lim _{n \rightarrow \infty} P^{*}\left(b_{n}\right)=\lim _{n \rightarrow \infty}\left\|\left(b_{n}\right)\right\|$ $=\lim _{n \rightarrow \infty}\left\|\hat{a}_{n}\right\|$. q.e.d.

THEOREM 5.11. If $\hat{S} / N_{p *}$ is a Banach space for every regular outer measure then $S$ is perfect. 
Proof. Given $a_{n}$ such that $a_{n} \wedge a_{m}=0$ for $n \neq m$ one defines $P\left(b+\sum_{\text {finite }} \lambda_{n} a_{n}\right)$ $=\sum \lambda_{n} / n^{2}$ where $b \perp a_{n}$ for all $n$. Let $P^{*}$ be the greatest outer measure compatible with $P$.

(a) $b+\Sigma \lambda_{n} a_{n} \geqq 0$ iff $b \geqq 0$ and $\lambda_{n} \geqq 0$ for all $n$.

Applying the operators $\left[a_{n}\right]$ and $[b]$ gives us this result.

(b) If $a \geqq 0$ and $P^{*}(a)=0$ then $a \perp a_{n}$ for all $n$.

Let $N$ be fixed. Then $0 \leqq P^{*}\left(\left[a_{N}\right] a\right) \leqq P^{*}(a)=0$.

Therefore for any $\varepsilon>0$ we can find $b_{m}, \lambda_{n}^{m}$ such that $\lim _{m \rightarrow \infty} \Sigma \lambda_{n}^{m} / n^{2} \leqq \varepsilon$ and

$$
\left(b_{m}+\Sigma \lambda_{n}^{m} a_{n}\right) \wedge\left[a_{N}\right] a \uparrow\left[a_{N}\right] a .
$$

Using the relation (a) one sees that $\lambda_{n}^{m} / n^{2} \uparrow_{m} \lambda_{n} / n^{2}$ and $\lim _{m} \sum \lambda_{n}^{m} / n^{2}=\Sigma \lambda_{n} / n^{2}<\varepsilon$. If $n \neq N$, applying [ $\left.a_{n}\right]$ to $\left(^{*}\right)$ we have $\lambda_{n}^{m} a_{n} \wedge 0 \uparrow 0$, which implies $\lambda_{n} a_{n} \wedge 0=0$. Thus $\lambda_{n} \geqq 0$. Therefore $\lambda_{N} / N^{2}<\varepsilon$ and applying $\left[a_{N}\right]$ to $\left(^{*}\right)$ we have $\lambda_{N}^{m} a_{N} \wedge$ $\left[a_{N}\right] a . \uparrow\left[a_{N}\right] a$ which implies $\lambda_{N} a_{N} \wedge\left[a_{N}\right] a=\left[a_{N}\right] a$.

Thus $\left[a_{N}\right] a \leqq \lambda_{N} a_{N} \leqq \varepsilon N^{2} a_{N}$ which implies that $\left[a_{N}\right] a=0$, that is, $a_{N} \cdots a$.

Let $s_{n}=\sum_{k=1}^{n} a_{k}$. $\hat{s}_{n}$ is a Cauchy sequence and thus $\lim _{m} \hat{s}_{n}=\hat{s}$ in norm. Therefore $\hat{s}_{n} \uparrow \hat{s}$. We may assume that $s$ is a positive representative of $\hat{s}$. We can find $b_{n}$ such that $P^{*}\left(\left|b_{n}\right|\right)=0$ and $s_{n} \leqq s+b_{n}$. Applying $\left[s_{n}\right]$ one has $s_{n} \leqq\left[s_{n}\right] s$ $+0 \leqq s$. Therefore $\sum_{n=1}^{\infty} a_{n}$ converges. q.e.d.

\section{BIBLIOGRAPHY}

1. I. Amemiya, A generalization of Riesz-Fisher's theorem, J. Math. Soc. Japan 5 (1953), 353-354.

2. G. Birkhoff, Lattice theory, 2nd ed., Amer. Math. Soc. Colloq. Publ. Vol. 25, Amer. Math. Soc., Providence, R. I., 1948.

3. N. Bourbaki, Intégration. Éléments de mathématique, Livre VI, Hermann, Paris, 1952.

4. H. Nakano, Modulared semi-ordered linear spaces, Maruzen, Tokyo, 1950.

5. —-, Modern spectral theory, Maruzen, Tokyo, 1950.

6. —- Teilweise geordnete Algebra, Japan. J. Math. 17 (1941), 425-511.

7. - Über Erweiterungen von allgemein teilweisegeordneten Moduln. II, Proc. Imp. Acad. Tokyo 19, (1943), 138-143.

8. M. H. Stone, Notes on integration. I, Proc. Nat. Acad. Sci. U.S.A. 34 (1948), 336-342.

\section{WAYNe STATE University,}

Detroit, Michigan 